\title{
Elektronische Rechnungen zur Optimierung der Financial Supply Chain: Status Quo, empirische Ergebnisse und Akzeptanzprobleme
}

\author{
Harald Schömburg, Michael H. Breitner \\ Institut für Wirtschaftsinformatik, Leibniz. Universität Hannover, \\ Königsworther Platz 1, 30167 Hannover \\ \{schoemburg|breitner\}@iwi.uni-hannover.de
}

\section{Einleitung}

In den vergangenen Jahren haben Unternehmen durch betriebswirtschaftliche Optimierungsprozesse vor allem in den produzierenden Bereichen große Einsparpotentiale erschlossen. Konzepte wie „Lean Production“, „Total Quality Management" oder auch „Business Process Reengineering“ werden bereits seit Anfang der 1990er Jahre erfolgreich eingesetzt und führen zu einer höheren Prozesseffizienz entlang der physischen Supply Chain.

Darüber hinaus erkennen die Unternehmen vermehrt die hohe Bedeutung des schnellen, kostengünstigen und präzisen Austauschs von Geschäftsdaten, um die Rationalisierungsmaßnahmen effektiv einsetzen zu können. So können Unternehmen im täglichen Geschäftsverkehr Effizienzgewinne dann erzielen, wenn sie die verschiedenen Geschäftsprozesse wie Warenbestellung, Lieferavisierung, Fakturierung und Zahlungsausgleich durchgehend automatisieren. Die Unternehmenskommunikation wird daher zunehmend (sowohl intern als auch extern) über elektronische Medien geführt. Die Vermeidung von fehleranfälligen Medienbrüchen trägt darüber hinaus zu weiteren Kosteneinsparungen bei. (AWV 2006, S. 5)

Die aktuell andauernde Wirtschafts- und Finanzkrise zwingt die Unternehmen jedoch weiter die Profitabilität durch Effizienzsteigerungen aufrecht zu halten oder gar zu erhöhen, um im Wettbewerb bestehen zu können. Vermeidung von negativen Auswirkungen auf Unternehmenswert und Aktienkurs, sowie die mittel- und langfristige Sicherung von Liquidität haben dabei höchste Priorität. Der Liquiditätsdruck wird zudem dadurch erhöht, da Banken und der Kapitalmarkt nicht mehr so leicht Kapital zur Verfügung stellen, wie dieses noch in der jüngsten Vergangenheit der Fall gewesen ist.

In den Fokus der Betrachtungen rückt daher die Optimierung der Financial Supply Chain, in welche neue Techniken und kostensparende Verfahren bisher nur ver- 
gleichsweise langsam Einzug hielten (Pfaff et al. 2004a, S. 107ff). Durchschnittlich 40 Prozent der „Finance \& Accounting Ressourcen“ sind daher auch heute noch durch Geschäftsprozesse ohne strategischen Charakter gebunden. (Langmack und Chalons 2006, S. 17) Rechnungsstellung, Rechnungseingang und Rechnungsbearbeitung gehören zu den Kernaufgaben der Finanzabteilungen. Aber gerade hier sind die Prozesse oft kleinteilig und von manuellen Tätigkeiten bestimmt. (Aleksic o.J., S. 1) Vor diesem Hintergrund ist es sehr wichtig, traditionelle Prozesse und Strukturen nicht nur zu hinterfragen, sondern im Rahmen von Optimierungsmaßnahmen unter Zuhilfenahme neuster Technologien aufzubrechen und umzustrukturieren. ${ }^{1}$

Methodisch wird in diesem Aufsatz wie folgt vorgegangen: zuerst wird eine umfangreiche, explorative Studie, welche die Ermittlung des Status Quo in der Rechnungseingangsbearbeitung deutscher Unternehmen zum Ziel hat, vorgestellt. Der Fokus wird hierbei insbesondere auf die Verbreitung und Akzeptanz der elektronischen Rechnung gelegt. Die Ergebnisse werden anschließend analysiert und diskutiert.

\section{Potentiale der elektronischen Rechnung}

Jährlich werden in Deutschland ca. sechs Milliarden Rechnungen verschickt; jedoch weitaus überwiegend auf dem traditionellen Postweg. Bei betriebswirtschaftlichen Kosten von ca. fünf bis sieben Euro pro versandter und rund zehn bis zwölf Euro pro empfangener Rechnung liegt hier ein Einsparpotential in Milliardenhöhe vor. (Schömburg et al. 2008, S.1ff)

Für die Seite des Versenders ist dies unmittelbar einleuchtend: im Bereich der Erstellung, der Ausgabe und des Versands von Rechnungen entstehen erhebliche Kosten für Material-, Porto- sowie Archivierung, die zu minimieren sich lohnt. Hinzu kommen Kosten für manuelle Prozesse sowie für Botengänge zur Post.

Im Idealfall erfolgt die Erstellung der Rechnung bereits automatisch nach Eingang und Prüfung einer Bestellung aus dem ERP-System heraus. Gleichwohl kann die Rechnungsstellung abhängig vom Automatisierungsgrad jedoch bis zu sechs Tage dauern, wobei anschließend die Postlaufzeiten hinzuzurechnen sind. (Pfaff et al. 2004b, S. 135ff) Hinzu kommt, dass elektronische Rechnungen auch vor Verlust geschützt sind; immerhin gehen ca. 0,5 Prozent aller Papierrechnungen beim Durchlaufen der Prozesse verloren. (Dörflinger 2005)

Interessant ist die elektronische Rechnungsstellung jedoch insbesondere für ein empfangendes Unternehmen, da die eingehenden Rechnungen vom Zeitpunkt des Eingangs bis zur Zahlung und Archivierung ein wesentlich breiteres Spektrum an Prozessen durchlaufen (siehe auch Abbildung 1). Das Öffnen und Sortieren der Eingangspost, die interne Verteilung der Rechnungen per Hauspost sowie die Er-

${ }^{1}$ Siehe vertiefend He und Cao 2009. 
fassung der Rechnungsdaten von Hand sind nur beispielhafte Prozesse, welche die Zeit zum Be- und Verarbeiten einer eingegangenen Papierrechnung gravierend erhöhen. So benötigt eine Rechnung, die ohne Einsatz geeigneter Software bearbeitet wird, rund 41 Tage bis zur Bezahlung. (Wuschech 2005, S. 1) Die lange Dauer der Bearbeitung hat zudem zur Folge, dass einzelne Abteilungen keinen vollständigen Überblick haben, wo eine Rechnung auf dem internen Postweg sich gerade befindet. So kann in der Praxis z. B. alleine die Kontrolle mit anschließender Genehmigung mehr als 14 Tage in Anspruch nehmen. (Wuschech 2005, S. 1 und S. 3)

Der Versand einer signierten Dokumentendatei alleine, wie z. B. PDF, erfültt jedoch das Kriterium einer elektronischen Rechnung nur im weiten Sinne. Der eigentliche Nutzen entsteht durch das Austauschen einer Datei mit definierten und strukturierten Inhalten wie z. B. einer XML-Datei, welche ohne manuelle Tätigkeiten automatisiert weiterverarbeitet werden kann. Hierdurch entstehen Einsparmöglichkeiten zwischen 70 und 90 Prozent der Gesamtkosten. (Schömburg et al. 2008, S. 19ff) Gleichzeitig wird durch die Verarbeitung ausschließlich elektronischer Daten und dem Einsatz automatisierter elektronischer Kontrollverfahren auf beiden Seiten die Transparenz sowie die Qualiät der Geschäftsprozesse gesteigert. (Frick 2003, S. 322)

Das Versenden einer Dokumentendatei ist optional und hat seinen eigentlichen Nutzen in den Fällen, in denen die Rechnungskontrolle nicht automatisch erfolgen kann. In solchen Fällen wird für die manuelle Verarbeitung eine leicht lesbare Ansicht benötigt, die darüber hinaus dem typischen Abbild einer Rechnung entspricht. (Tanner und Wölfle 2005, S. 7)

Wie die folgende Abbildung verdeutlicht, erlaubt die elektronische Rechnung eine Optimierung nahezu des kompletten Geschäftsprozesses.

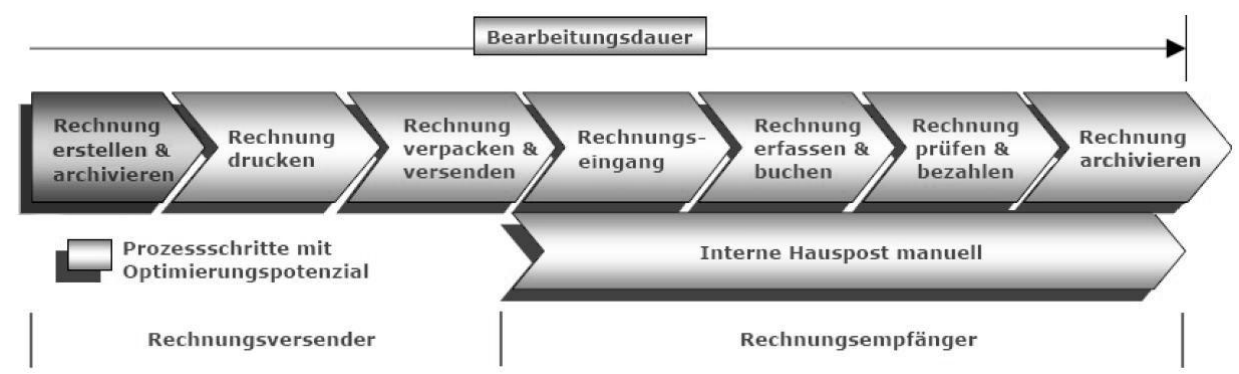

Abbildung 1: Optimierungspotentiale durch elektronische Rechnungsstellung 
Auf eine detaillierte Beschreibung weiterer Vorteile sei an dieser Stelle verzichtet. Stattdessen soll die nachfolgende Auflistung das Potential sowie die wesentlichen Vorteile verdeutlichen. ${ }^{2}$ (Schömburg und Breitner 2008, S. 17)

- Keine Porto- und Materialkosten

- Zahlungsfristen exakt, da lediglich Sekunden bis zur Zustellung

- Garantierte Zustellung mit erleichtertem Nachweis

- Beschleunigte Durchlaufzeiten, einschl. Rechnungsprüfung und Freigabe

- Dadurch Wegfall von Skontoverlusten aufgrund zu langer Durchlaufzeiten

- Keine Medienbrüche mehr sowie dadurch wegfallende Mehrfachablage

- Verringerter Personaleinsatz

- Wegfall manueller Fehler durch Automatisierung (insbesondere Fehlbuchungen)

- Schnell ersichtliche aktuelle Verarbeitungsstufe einzelner Rechnungen

- Wegfall des temporären physischen Verlusts von Rechnungen

- Vereinfachte und kostengünstigere Archivierung

- Deutlich verringerte Umweltbelastung

\section{Die empirische Studie}

\subsection{Forschungsfragen}

In den letzten Jahren wurden europaweit Richtlinien erlassen, die es erlauben, den Rechnungsversand nach neuen Regeln vollständig elektronisch über das Internet abzuwickeln. Diese Regelungen sind zurzeit Gegenstand einer Vielzahl kontroverser Diskussionen, jedoch erkennen inzwischen immer mehr Unternehmen, dass sich die notwendigen Anfangsinvestitionen lohnen und einen schnellen ROI liefern. Diese Ansätze und Entwicklungen in der Praxis werden durch die Literatur unterstützt. Es liegen bereits diverse Studien vor, die der elektronischen Rechnungsstellung ein enormes Einsparpotential zusprechen. ${ }^{3}$ Diese Studien belegen aber auch, dass die elektronische Rechnungsstellung bislang noch nicht so weit verbreitet ist, wie ihr praktisches Potential eigentlich nahe legt.

Allerdings sind die Studien oftmals populärwissenschaftlich gehalten und eine detaillierte sowie wissenschaftlich fundierte Analyse der elektronischen Rechnungsstellung - insbesondere im Hinblick auf die damit einhergehenden Akzeptanz- und Umsetzungsprobleme in der Praxis - ist bislang nur unzureichend erfolgt. Folglich stehen diesbezüglich zurzeit noch nicht genügend Literatur und Quellenmaterial zur Verfügung. Es scheint so, als ob eine verbreitete Euphorie

\footnotetext{
2 Risiken und hemmende Faktoren der elektronischen Rechnung werden in Kapitel 4.3 sowie in Kapitel 5 analysiert.

${ }^{3}$ Zur Vertiefung siehe (Schömburg und Breitner 2008, S. 19f).
} 
angesichts der tatsächlichen und potentiellen Einsparungen auf der Kostenseite die mit der Einführung verbundenen Umsetzungsprobleme verdrängt. Dabei wird häufig übersehen, dass die elektronische Rechnungsstellung ihr wirtschaftliches Potential nur dann voll entfalten kann, wenn der gesamte Prozess optimal in den unternehmerischen Ablauf integriert und von Akzeptanz getragen ist. Das gilt nicht nur für die externen Abläufe gegenüber den Geschäftspartnern, sondern ebenso für die internen Vorgänge.

Um der Aktualität des Themas gerecht zu werden und die Bedeutung der Forschungslücke verbunden mit dem dringenden Handlungsbedarf hervorzuheben, wurden die vorliegenden Expertenbefragungen durchgeführt. ${ }^{4}$

Im Vorfeld der Befragungen wurden folgende Forschungsfragen gestellt:

1. Wie ist der Status Quo in der papierbasierten Eingangsrechnungsbearbeitung hinsichtlich technologischer Lösungen?

2. Welche Zukunftsaussichten hat der elektronische Rechnungsempfang und welche Rolle spielen Outsourcing-Dienstleister dabei?

\subsection{Methodik und Vorgehensweise}

In der Zeit von September 2007 bis März 2009 wurden drei repräsentative Expertenbefragungen zum Thema „Rechnungseingangsbearbeitung“" durchgeführt. ${ }^{5}$ Die Explorationen unterscheiden sich in großen Teilen hinsichtlich der gestellten Fragen und beabsichtigen daher nicht, eine zeitliche Entwicklung darzustellen. Insgesamt lassen sich jedoch aus den jeweiligen Ergebnissen eindeutige Tendenzen herausarbeiten. ${ }^{6}$

\begin{tabular}{|c|c|c|}
\hline \multicolumn{3}{|c|}{ Literaturrecherche } \\
\hline$\downarrow$ & $\downarrow$ & $\downarrow$ \\
\hline $\begin{array}{l}\text { Empirische Analyse } 1 \\
\text { - } 2007 \\
\text { - Papierbasierte und } \\
\text { elektronische } \\
\text { Rechnungen } \\
\text { - } \mathrm{N}=50\end{array}$ & $\begin{array}{l}\text { Empirische Analyse } 2 \\
\text { - } 2008 \\
\text { - Elektronische } \\
\text { Rechnungen } \\
\text { - } \mathrm{N}=76\end{array}$ & $\begin{array}{l}\text { Empirische Analyse } 3 \\
\text { - } 2009 \\
\text { - Papierbasierte } \\
\text { Rechnungen } \\
\text { - } \mathrm{N}=85\end{array}$ \\
\hline
\end{tabular}

Abbildung 2: Vorgehen im Rahmen der empirischen Untersuchungen

\footnotetext{
4 Die drei Befragungen beziehen sich ausschließlich auf die Seite des Rechnungsempfängers, da dieser das größere Einsparpotential besitzt und seine Zustimmung bzw. Akzeptanz unabdingbare gesetzliche Voraussetzung für das elektronische Verfahren ist.

${ }^{5}$ Die Gestaltung und Durchführung der Befragungen wurde durch die sgh Service AG, Hildesheim, einem der größten unabhängigen Full-Service-Dienstleister für Outsourcing im Rechnungswesen in Deutschland, praxisnah unterstützt. Siehe www.sgh-net.de.

${ }^{6}$ Die jeweiligen Fragebögen der Analysen 1 und 2 sowie eine ausführliche Besprechung der Ergebnisse finden sich in (Schömburg und Breitner 2008) sowie (Schömburg et al. 2008). Die dritte Analyse wurde noch nicht veröffentlicht.
} 
Die erste Befragung im Jahr 2007 wurde im Rahmen einer Diplomarbeit durchgeführt. Ihr Schwerpunkt lag vor allem im Aufdecken der Problemfelder der papierbasierten Rechnungsbearbeitung sowie der Verbreitung und Akzeptanz der elektronischen Rechnung. Aufbauend auf den gewonnen Erkenntnissen wurden Akzeptanz und Potentiale der elektronischen Rechnung in einer zweiten Befragung vertiefend analysiert. Die dritte Untersuchung hatte die detaillierte Analyse der Problemfelder der papierbasierten Rechnungsbearbeitung zum Ziel.

Im Rahmen der drei Untersuchungen wurden diejenigen Personen als Experten ausgewählt, die sich detailliert mit der Rechnungsbearbeitung des jeweiligen Unternehmens auskennen. Überwiegend handelte es sich hierbei um Sachbearbeiter des Rechnungswesens, gelegentlich wurde jedoch auch an den Leiter der Abteilung weitergeleitet, wenn die Fragen als „zu detailliert“ empfunden wurden.

Anhand der zweiten Expertenbefragung soll exemplarisch das methodische Vorgehen sowie die Repräsentativität der Stichprobe vertiefend veranschaulicht werden. Die übrigen Befragungen weisen ein analoges Schema auf.7

Die zweite Befragung fand im Zeitraum Juni bis August 2008 statt. Insgesamt wurden 76 Experten zur Rechnungseingangsbearbeitung des jeweiligen Unternehmens befragt, wobei 50 von ihnen telefonisch, die anderen 26 Teilnehmer online befragt wurden.

Zur Ermittlung der jeweiligen Telefonteilnehmer wurde eine Liste des Instituts für Mittelstandsforschung Bonn aus dem Jahr 2007 herangezogen. (IfM 2007) Als Erhebungsgrundlage wurden die jeweils zehn umsatzstärksten Unternehmen der jeweiligen Bundesländer ermittelt, wobei die einzelnen Kandidaten nach alphabetischer Reihenfolge der Bundesländer und der Höhe ihrer Umsätze kontaktiert wurden. Die telefonische Befragung endete nach Mitarbeit des fünfzigsten Teilnehmers. Die zentralen Kontaktdaten wurden aus dem Impressum der WWW-Seiten entnommen. Es wurde direkt nach der Kreditorenbuchhaltung verlangt.

Die Online-Befragung fand parallel zur telefonischen Erhebung statt. Hierfür wurde der für die telefonische Befragung entworfene Fragebogen in ein OnlineGegenstück übertragen. Die Ausarbeitung erfolgte durch die Firma index „Agentur

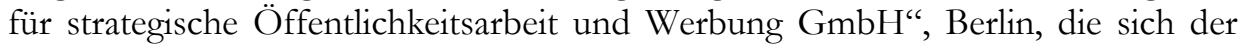
Software polliscope ${ }^{8}$ bediente. Die Kandidaten wurden anhand einer zufälligen Stichprobe von Unternehmen aus den Branchen Industrie und Handel ermittelt. Der Verweis zum Online-Fragebogen wurde mittels E-Mail versandt, welche zusätzlich erläuternde Informationen zu dem laufenden Forschungsprojekt beinhaltete. Darüber hinaus wurde um die Weiterleitung an einen zuständigen Mitarbeiter aus der Kreditorenbuchhaltung gebeten. Die Zeit zum Ausfüllen des Fragebogens war auf ca. zehn Minuten bemessen.

\footnotetext{
${ }^{7}$ Die Fragebögen sind auf Anfrage bei den Autoren erhältlich.

8 Siehe www.polliscope.de.
} 
Im Folgenden werden ausgewählte Ergebnisse der empirischen Untersuchungen vorgestellt. Die Darstellung erfolgt hierbei nicht in zeitlicher, sondern in sachlogischer Reihenfolge.

\section{Ausgewählte Ergebnisse}

\subsection{Struktur des Rechnungseingangs}

Die Unternehmen wurden nach der Struktur ihres Rechnungseingangs befragt. Mit 87 Prozent dominiert der Empfang per Post. Die am zweithäufigsten genannte Antwort „EDI“ liegt mit 7 Prozent bereits deutlich zurück. Der Rechnungsempfang über E-Mail mit Signatur beträgt 1 Prozent. Bemerkenswert ist, dass der Rechnungsempfang per E-Mail ohne Signatur 2 Prozent ausmacht. Viele Unternehmen gaben im Gespräch an, die Rechnungen jedoch auf dem Postweg oder per Fax nachzufordern. Oftmals werden die Rechnungen allerdings einfach akzeptiert.

Die Rubrik „Sonstige“ umfasst neben Rechnungen per Fax auch die Inanspruchnahme von Dienstleistern.
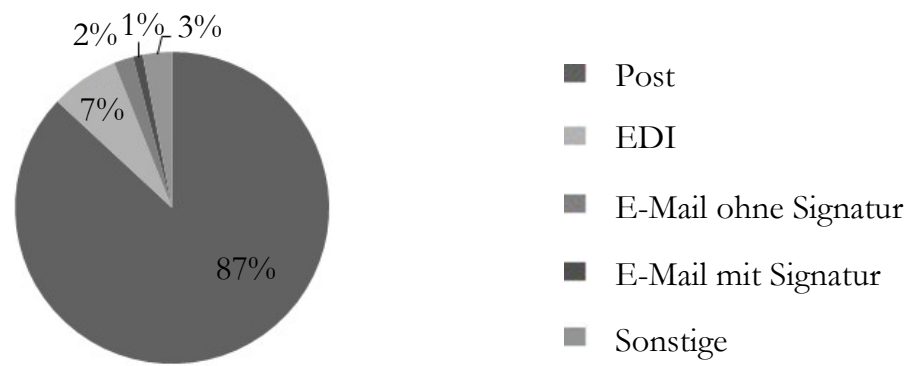

Abbildung 3: Struktur des Rechnungseingangs 2008, N = 72

\subsection{Handhabung papierbasierter Eingangsrechnungen}

Die Unternehmen wurden gefragt, wie sie die papierbasierten Eingangsrechnungen bearbeiten. Eine vollständig manuelle Bearbeitung erfolgt in 55 Prozent der Fälle. Scanlösungen sind in 55 Prozent der Unternehmen vorhanden, jedoch werden diese zu 51 Prozent ausschließlich für die elektronische Archivierung genutzt; vereinzelt wurde jedoch auch ein „frühes“ Scannen9 vollzogen. Optical Character Recognition (OCR) wird in 27 Prozent genutzt, weitere 2 Prozent beziehen einen Dienstleister zur Rechnungsbearbeitung ein. In der Gesamtbetrachtung erfassen somit $71 \%$ ihre Rechnungen von Hand.

\footnotetext{
${ }^{9}$ Beim „frühen“ Scannen wird ein Foto (kein Datensatz) der Rechnung erzeugt, welches elektronisch an die jeweiligen Abteilungen weitergeleitet wird. Hausinterne Botengänge entfallen dadurch.
} 


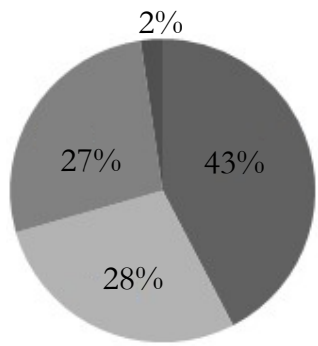

- Manuell

- Scannen ohne OCR

- Scannen mit OCR

- Dienstleister

Abbildung 4: Formen der Bearbeitung von Papierrechnungen 2009, N = 85

Teilnehmer, die ihre Rechnungen nicht per OCR erfassen, wurden gefragt, ob sie eine Bearbeitung per OCR gegenüber der manuellen Erfassung generell bevorzugen würden. Dies wurde von 72 Prozent bejaht. 8 Prozent äußerten sich unentschlossen - i.d.R. mangels ausreichender Kenntnisse über diese Technologie.

Dessen ungeachtet gaben Unternehmen, die bereits OCR verwenden, zu 55 Prozent an, den anschließenden manuellen Korrekturaufwand als akzeptabel zu sehen. Positiv äußerten sich insgesamt 27 Prozent, die den Korrekturaufwand als gering (18 Prozent) oder als sehr gering (9 Prozent) einschätzen. 18 Prozent der befragten OCR-Anwender sehen ihn jedoch als problematisch an.

\subsection{Akzeptanz und Hürden der elektronischen Rechnung}

Analog zu der Frage, ob OCR einer manuellen Rechnungsbearbeitung vorzuziehen sei, wurden die Teilnehmer gefragt, ob sie generell eine vollständig elektronische Rechnung bevorzugen würden. 70 Prozent bejahten diese Frage. 5 Prozent gaben sich unentschlossen mit Verweis auf ihren mangelnden Kenntnisstand.

Unternehmen, welche die Frage verneinten, wurden nach den Gründen gefragt. Die Antworten brachten jedoch keine neuen Erkenntnisse und entsprachen im Wesentlichen den Hinderungsgründen einer Einführung allgemein (s. Abb. 5).

Die Umfrage ergab ferner, dass insgesamt 23 Prozent der Befragten den elektronischen Rechnungsempfang bereits praktizieren (11 Prozent) oder eine Einführung ernsthaft beabsichtigen (12 Prozent). Allerdings ist der Einsatz meist noch auf Testprojekte oder ausgewählte Lieferanten beschränkt. Eine Anwendung in großem Umfang hat folglich noch nicht stattgefunden. Hinzu kommt, dass auch ein rechnungsbegleitender Datensatz in der Regel nicht genutzt wird.

Die übrigen Teilnehmer, die noch keinen elektronischen Rechnungsempfang einzuführen beabsichtigen, empfinden insbesondere die Integration in den Workflow als hinderlich (33 Prozent). Weitere 26 Prozent sehen schlichtweg keinen Bedarf und ebenfalls 26 Prozent bemängeln das Desinteresse an der elektronischen Rechnungsstellung auf Seiten der Rechnungssteller. 


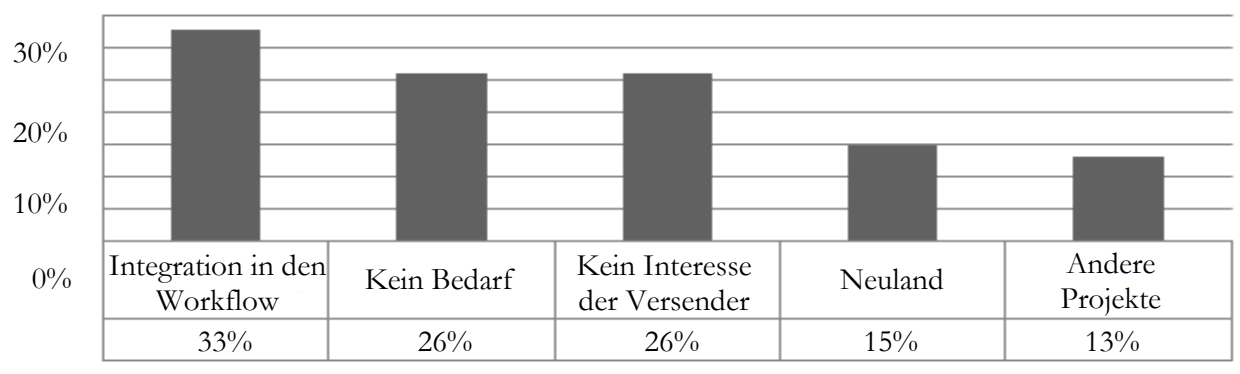

Abbildung 5: Haupthinderungsgründe einer Einführung des elektronischen Rechnungsempfangs 2008, $\mathrm{N}=46$; Mehrfachnennungen möglich

\subsection{Bereitschaft zum Auslagern}

Die Unternehmen wurden abschließend gefragt, ob sie sich vorstellen könnten, die Rechnungsbearbeitung insgesamt (d.h. elektronisch und papierbasiert) an einen Dienstleister auszulagern. Dies stellt für 71 Prozent keine Option dar. 6 Prozent zeigten sich unentschlossen.

Nach den Gründen für die Ablehnung gefragt, äußerten 31 Prozent Bedenken, ob ein Außenstehender überhaupt in der Lage sein kann, die unternehmensindividuellen Abläufe sachgerecht abzubilden. An zweiter Stelle stand die Unternehmensphilosophie, die eine Verlagerung an einen Dritten generell ausschließt (22 Prozent). Ferner wird es kritisch gesehen, dass ein externer Dienstleister über sensible Rechnungsdaten - im Zweifel auch der Konkurrenten - verfügt (17 Prozent).

\section{Diskussion und Ausblick}

Die Ergebnisse der Expertenbefragungen zeigen, dass die elektronische Rechnungsstellung noch längst nicht die Verbreitung gefunden hat, die ihr praktisches Potential nahelegt. Dies bestätigt ebenfalls eine internationale Studie der EUKommission aus dem Jahr 2007, welche rechtliche Unsicherheit, Skepsis gegenüber der Automatisierung sowie eine fehlende Standardisierung als Hinderungsgründe aufführt. (European Commission 2007, S. 10ff)

Wie die Ergebnisse der Expertenbefragungen jedoch zeigen, sind die Hürden der elektronischen Rechnung allein aus deutscher Sicht differenzierter zu betrachten und liegen in verschiedenen sich zum Teil überlagernden Gründen.

Rechtliche Bedenken wurden im Rahmen der Expertenbefragungen nicht direkt geäußert. Allerdings sehen die Unternehmen Probleme, die elektronische Rechnung organisatorisch sowie rechtlich in den aktuellen Workflow zu integrieren. Zwar ist das Thema der elektronischen Rechnungsstellung inzwischen so weit „gereift", dass zahlreiche gute und einfach anzuwendende Lösungen am Markt existieren, allerdings mangelt es an einem verlässlichen und günstigen Standard. 
Hieraus resultiert ferner eine „Henne-Ei-Problematik”, wie sie für neue Kommunikationsformen typisch ist. Um diese zu lösen, bedarf es jedoch mehr als nur einer Standardisierung, da das Potential der elektronischen Rechnungsstellung in Deutschland nicht hinreichend bekannt ist. Allerdings ist zu erwarten, dass sich dies im Laufe der Zeit ändern wird: mehr und mehr wird das Potential der elektronischen Rechnung auch im Vergleich zu klassischen Prozessen analysiert und veröffentlicht. Darüber hinaus geraten die Verwaltungskosten der Unternehmen zunehmend in den Fokus. (Siehe z. B. Frey et al. 2008)

Entscheidend ist jedoch vor allem der fehlende Ansporn der Rechnungsempfänger durch die Rechnungssteller, das fehlende Fachwissen sowie die erforderlichen, umfangreichen Investitionen bspw. in Signatur-Soft- und -Hardware oder ein elektronisches Archiv.

Die Rechnungssteller für den elektronischen Versand zu gewinnen, stellt aktuell eine große Hürde dar, weil im Rechnungsausgang das Einsparpotential wesentlich geringer ausfällt als im Rechnungseingang. Außerdem können i.d.R. nicht alle Empfänger per elektronischer Rechnung erreicht werden, so dass der Versender gefordert ist, einen zweiten parallelen Workflow bereitzustellen. Hierdurch entstehen ihm zusätzliche Kosten.

Wird jedoch erst einmal eine gestiegene Anzahl Rechnungen elektronisch verschickt, tragen Netzeffekte dazu bei, dass sich die genannten hohen Investitionskosten auf beiden Seiten innerhalb kurzer Zeit amortisieren und der Nutzen der Investition kontinuierlich zunimmt. Es bleibt allerdings zu beachten, dass die Aneignung des notwendigen Fachwissens weiterhin einen hohen Aufwand darstellt. Die Zahl der in Deutschland zu beachtenden Regelungen und Gesetze ist hoch (z. B. AO, GDPdU, GoBS, SigG, UStG). Aus ihnen resultiert eine umfangreiche Liste von Anforderungen, die an die einzusetzende Hard- und Software sowie an ihren Betrieb gestellt werden.

Als personalintensiver Bereich der weder das Kerngeschäft des Unternehmens noch der Buchhaltung darstellt, empfiehlt sich alternativ ein Auslagern der Rechnungsbearbeitung an externe Dienstleister. Neben der Realisierung von Kosteneinsparungen durch Skaleneffekte, ermöglichen sie die notwendigen Investitionskosten zu minimieren und verfügen zusätzlich über das nötige Fachwissen und Erfahrungen mit den technischen und rechtlichen Grundlagen. Dennoch: die Bereitschaft der Befragten zum Outsourcing hält sich in Grenzen.

Ein Grund hierfür ist die derzeit noch geringe Entwicklung des „Business Process Outsourcing (BPO) Marktes”: es findet sich lediglich ein ausgeprägter Zweig speziell für Finanzdienstleister. Die übrigen Bereiche wie bspw. BPO für Finance \& Accounting sind derzeit nur in Ansätzen vorhanden. (Gross et al. 2006, S.55)

Dennoch, oder gerade deshalb, zeichnet sich insbesondere der deutsche Markt auf diesem Gebiet durch hohes Wachstumspotential aus (Georgius und Heinzl 2005, S. $409 \mathrm{f}$ und S. 425), so dass mittelfristig mit einer Verbesserung der Dienstleistungsangebote $\mathrm{zu}$ rechnen ist. 
Ungeachtet dessen wird es aus heutiger Sicht jedoch nur eine Frage der Zeit sein, bis die elektronische Rechnung sich weitflächig durchsetzen wird. Allerdings bleibt vorerst offen, inwieweit diese „neue" Form der Rechnungsstellung die „traditionellen" Formen ersetzen wird. Nicht für jedes Unternehmen kommt die elektronische Rechnungsstellung als alleiniges Verfahren in Betracht. Vielmehr wird oftmals weiterhin ein begleitender Prozess in Papierform bestehen, z. B. aufgrund von Geschäftsbeziehungen zum außereuropäischen Ausland oder aufgrund ständig wechselnder „,kleinerer" Geschäftspartner, bei welchen die Anpassung der Schnittstellen und Datensätze in keinem Verhältnis zum Nutzen steht.

Selbst unter Berücksichtigung dieser Problemfelder bleibt das Verbesserungspotential bei den Prozessen - insbesondere im Hinblick auf die Kosten - und den Auswirkungen auf Umwelt und Ressourcen beachtlich und ist als ein lohnenswertes Ziel anzustreben. ${ }^{10}$

\section{Literatur}

Aleksic D (o.J.) Damit die Rechnung aufgeht. http://www.fibu markt.de/Fachinfo /ERP-Software/Damit-die-Rechnung-aufgeht.html. Abruf am 2009-29-08.

AWV (2006) Der elektronische Rechnungsaustausch - Ein Leitfaden für Unternehmen zur Einführung elektronischer Rechnungen. AWV, Eschborn.

Dörflinger U (2005) Bei Rechnungen lässt sich sparen. http://www.computer woche.de/heftarchiv/2005/49/1207366/. Abruf am 2009-08-29.

European Commission Informal Task Force on e-Invoicing (2007) European Electronic Invoicing (EII) Final Report.

http://ec.europa.eu/information_society/ eeurope/i2010/docs/studies/eei3.2-e-invoicing_final_report.pdf. Abruf am 2009-23-11.

Frey S, Habbel R, Heinz I (2008) Verwaltungskosten: Unternehmen lassen Einsparpotenziale ungenutzt - Eine Umfrage unter den Finanzchefs von TopUnternehmen in Deutschland, Österreich und der Schweiz. Booz \& Company, Berlin.

Frick B (2003) Digitale Signaturen und Zeitstempel im Archiv. In: Digitale Signaturen in der Praxis - Leitfaden zur Prozessoptimierung und Kostenreduktion in Unternehmen und Behörden, AWV, Eschborn.

Georgius A und Heinzl A (2005) Strategien und Erfolgsfaktoren von Anbietern im IT und Business Process Outsourcing. In: Ferstl OK, Sinz EJ, Eckert S, Isselhorst T (Hrsg) Wirtschaftsinformatik 2005: eEconomy, eGovernment, eSociety, Physica-Verlag, Heidelberg.

${ }^{10}$ Siehe vertiefend Penttinen et al. 2008. 
Gross J, Bordt J, Musmacher M (2006) Business Process Outsourcing: Grundlagen, Methoden, Erfahrungen. Gabler, Wiesbaden.

He Y, Cao Y (2009) The New Trend of Financial Process Reengineering: Financial Shared Services. In: Proceedings of the 2009 Second International Symposium on Electronic Commerce and Security - Volume 01, IEEE Computer Society, Washington.

Langmack J, Chalons C (2006) Business Process Outsourcing (BPO) in Deutschland. EDS, Rüsselsheim.

IfM (2007) Die volkswirtschaftliche Bedeutung der Familienunternehmen; Top 500-Liste: Sortierung nach Bundesländern und alphabetischer Reihenfolge. Ifm, Bonn.

Pentinnen E, Hallikainen P, Salomäki T (2008) Impacts of the Implementation of Electronic Invoicing on Buyer-Seller Relationships. In: Proceedings of the 42nd Hawaii International Conference on System Sciences, IEEE Computer Society, Washington.

Pfaff D, Skiera B, Weitzel T (2004a) Financial-Chain-Management - Ein generisches Modell zur Identifikation von Verbesserungspotenzialen. WIRTSCHAFTSINFORMATIK 46(2):107-118.

Pfaff D, Skiera B, Weiss J (2004b) Financial Supply Chain Management. Galileo, Bonn.

Schömburg H, Breitner MH (2008) Elektronische Rechnungsstellung: Prozesse, Einsparpotentiale und kritische Erfolgsfaktoren. In: IWI Diskussionsbeiträge \#21, Institut für Wirtschaftsinformatik Leibniz Universität Hannover.

Schömburg H, Hoppen G, Breitner MH (2008) Expertenbefragung zur Rechnungseingangsbearbeitung: Status quo und Akzeptanz der elektronischen Rechnung. In: IWI Discussion Paper \# 27, Institut für Wirtschaftsinformatik Leibniz Universität Hannover.

Tanner C, Wölfle R (2005) Elektronische Rechnungsstellung zwischen Unternehmen - Konzentriertes Wissen aus der swissDIGIN-Initiative zur Förderung des elektronischen Rechnungsaustauschs. FHBB, Basel.

Wuschech F (2005) Das Ende der Papierstapel. http://www.pcwelt.de/knowhow/business/569894/. Abruf am 2009-08-29. 